\title{
THE EFFECT OF A VEHICLE CONTROL DEVICE ON DRIVER PERFORMANCE IN A SIMULATED TANK DRIVING TASK
}

\author{
Ellen C. Haas \\ U.S. Army Research Laboratory \\ Human Research and Engineering Directorate \\ Aberdeen Proving Ground, Maryland, USA \\ E-mail: ehaas@arl.army.mil \\ Micaela Kunze \\ Bundesamt für Wehrtechnik und Beschaffung \\ Postfach 7360 \\ 56057 Koblenz, Germany \\ E-mail: MicaelaKunze@bwb.org
}

Summary: The purpose of this study was to determine the effect of different vehicle controllers on driver performance in a simulated tank driving task. Eight male civilian volunteers with normal visual acuity drove a simulated tank on a digitized road terrain. The subject monitored his speed by means of a speedometer shown on the monitor. Independent variables were driving controller (joystick, or steering wheel with attached brake and accelerator pedal), and assigned driving speed of 15 or $45 \mathrm{mph}$ (the maximum speed at which the subject was permitted to travel). Dependent variables were mean driving speed (the average speed at which the subject actually drove), and the proportion of time the center of the vehicle remained on the road during travel. Results indicated that subjects using the steering wheel obtained a significantly greater mean driving speed than those using the joystick only when they were permitted to drive a maximum speed of $45 \mathrm{mph}$. This difference may have little practical significance because the mean driving speed for the two controllers differed by less than $5 \mathrm{mph}$. There was no significant difference between controllers for the proportion of time the driver was able to keep the center of the vehicle on the road. Results implied that joystick controls have potential as an alternative control technology, and that the ergonomic placement of the joystick could be an important factor in enhancing driver performance.

\section{INTRODUCTION}

Few researchers have addressed issues relating to the use of the joystick in ground vehicle applications. In the choice of design of future Army ground vehicles, and in future robotics and teleoperation applications, drivers may potentially use joystick controllers rather than steering wheels. Joystick control provides several important benefits, including the advantage of utilizing the flexibility and dexterity of the human hand, wrist and fingers to increase the degree of driver control over vehicle operations. One researcher noted that if control and feedback parameters are chosen carefully, a joystick controller may potentially outperform a steering wheel with brake and accelerator pedals (Lee, 2000). The purpose of this study was to determine the effect of different vehicle controllers on driver performance in a simulated tank driving task. The results of this study will be used to develop new U.S. Army crewstation concepts, and to provide more knowledge for further studies such as the U.S. Army’s Crewstation Automation Testbed, as well as Army Future Scout and Future Combat Systems. 


\section{METHODOLOGY}

\section{Subjects, Facilities and Apparatus}

Subjects were eight, U.S. Army Department of Defense male, right-handed civilian volunteers solicited from the Human Research and Engineering Directorate (HRED) workforce for the purpose of participating in this experiment. Subjects were screened for normal visual functioning. The experiment was conducted at HRED, Building 459, Aberdeen Proving Ground, Maryland.

Apparatus included a Silicon Graphics (SGI) Indigo Workstation. The steering wheel device was a Thrustmaster NASCAR Model Pro Racing Steering wheel with pedal accelerator and brake. The joystick was a Logitech Wingman Joystick.

\section{Experimental Tasks}

In the driving task, the participant used the steering wheel or joystick device to drive on digitized road terrain presented on the SGI Indigo workstation monitor located directly in front of him. The participant used the steering wheel accelerator and brake on the floor to maintain speed, or manipulate the joystick device to accelerate, steer or brake (pushing the joystick forward and backward to furnish acceleration and braking action). The participant monitored his speed by means of a speedometer shown on the workstation monitor. The experimenter monitored the participant's speed during the experiment to ensure that the subject's speed remained at or close to the required speed for that session.

The terrain was a digitized version of the Aberdeen Test Center Demo II Course at Churchville, Maryland. Five different courses were used, one for the training sessions, and one for each of the four experimental sessions. The courses were approximately the same length and had the same number of turns.

Differing levels of driving task workload were manipulated by specifying different speeds at which the participant performed the driving task. The speeds, which were representatives of low- and high-workload tank driving conditions, were 15 and $45 \mathrm{mph}$. Driving speed and control apparatus were counterbalanced across all participants and experimental sessions to avoid practice effects.

\section{Procedure}

Prior to the beginning of the experiment, each subject was given a half-hour training session in which he was introduced to the driving task and the controller used in the first experimental session. The subject then drove a practice test course at $45 \mathrm{mph}$ for 30 minutes.

After a 30-minute break, the subject performed the experimental driving task at the predetermined conditions of driving speed (15 or $45 \mathrm{mph}$ ) and vehicle controller (joystick or steering wheel). He was asked to maintain the assigned speed and remain on the course while driving. When the driving task was completed, the participant filled out a NASA TLX workload measure and had a 30-minute break. After the break, the subject started the next experimental 
condition in which he drove at the remaining pre-determined driving speed. When the driving task was completed, the subject filled out a NASA TLX worked measure, and the subject was advised to return the next day.

The next day, the subject experienced one practice and two experimental sessions using a different steering device, at the pre-assigned driving speeds of 15 and $45 \mathrm{mph}$. When each driving task was completed, the subject again filled out a NASA TLX workload measure. When both experimental sessions were completed, the experiment ended.

\section{Experimental Design}

A 2 x 2 mixed factor, repeated measures design was used for data collection and to structure data analysis. Independent variables were driving controller (joystick, or steering wheel with attached brake and accelerator pedal), and assigned driving speed of 15 or $45 \mathrm{mph}$ (the maximum speed at which the subject was permitted to travel). Dependent variables were mean driving speed (the average speed at which the subject actually drove), and the proportion of time the center of the vehicle remained on the road during travel.

\section{RESULTS}

An Analysis of Variance (ANOVA) was performed for each dependent variable to determine whether statistically significant differences existed between main effects or interaction effects in the study. Significant effects $(\mathrm{p} \leq 0.05)$ were explored on a post hoc basis.

The ANOVA for mean driving speed indicated significant main effects for controller $(\underline{F}=7.24, \underline{p}$ $=0.031)$, for assigned driving speed $(\underline{F}=2130.84, \underline{p}=0.000$, and for the control $\mathrm{x}$ speed interaction $(\underline{F}=8.412, \underline{p}=0.023)$. The interpretation of the higher-order interaction precludes the interpretation of the lower-order interactions. The data for the controller $\mathrm{x}$ speed interaction, which is plotted in Figure 1, indicated that at assigned speeds of $15 \mathrm{mph}$, subjects obtained a mean driving speed of $14.7 \mathrm{mph}$ using a steering wheel, and a mean driving speed of $14.5 \mathrm{mph}$ using a joystick. This difference was not statistically significant ( $\mathrm{p}>0.05)$. At assigned speeds of $45 \mathrm{mph}$, subjects using a steering wheel obtained a mean driving speed of $39.9 \mathrm{mph}$, and 38.4 mph using a joystick. Although this difference was statistically significant $(\mathrm{p}<0.05)$, it may have little practical significance because it is less than $5 \mathrm{mph}$.

The ANOVA indicated no significant main effects or interactions for the proportion of time the driver was able to keep the vehicle on the center of the road. Thus, vehicle controller made no significant difference in the ability of subjects to maintain control of the vehicle.

Subject comments provided insight into the use of the joystick controller. Although some subjects commented that the steering wheel provided an advantage because of the large amount of prior experience use in the automobile, others noted that the joystick seemed to provide more control on curves. Some drivers commented that the placement of the joystick was important, and that arm support was needed during driving tasks in order to prevent driver fatigue. 
Figure 1: Mean Driving Speed as a Function of Assigned Speed

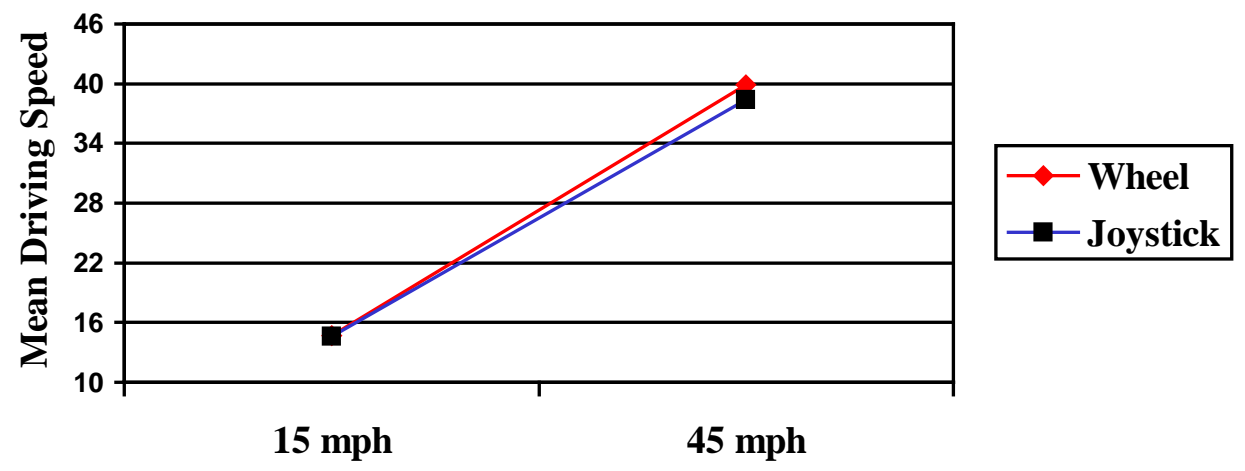

Assigned Driving Speed

\section{CONCLUSIONS}

The results indicate that the steering wheel provided no significant advantage over use of the joystick. At a relatively slow speed of $15 \mathrm{mph}$ there was no significant difference in mean driving speed between joystick and controller. At faster driving speed of $45 \mathrm{mph}$, the difference between joystick and controller was small enough to have no practical significance. In addition, controller type played no significant role in assisting drivers to keep their vehicle in the center of the road. However, joystick controls have potential as an alternative control technology because they provide more control over curves, possibly because they permit greater use of the hand, wrist and fingers to increase the degree of driver control.

Subject comments implied that the ergonomic placement of the joystick could be an important factor in enhancing driver performance. Subjects related that proper support of the hand, wrist and forearm are important to efficient and effective joystick use. Future research should explore proper joystick placement in the crewstation to allow optimum use in steering tasks.

\section{REFERENCES}

Lee, J.D. (2000). A summary of issues concerning joystick control of ground vehicles. Technical report prepared for Booz-Allen \& Hamilton Inc. Iowa City: Author. 